\title{
Vaccination with human induced pluripotent stem cells creates an antigen-specific immune response against HIV-1 gp160
}

\author{
Shinji Yoshizaki' , Mayuko Nishi' , Asami Kondo ${ }^{1}$, Yoshitsugu Kojima' ${ }^{1}$, Naoki Yamamoto $^{2}$ and Akihide Ryo ${ }^{1 *}$ \\ Department of Microbiology, Yokohama City University Graduate School of Medicine, Yokohama, Kanagawa, Japan \\ 2 Department of Microbiology, National University of Singapore, Singapore, Singapore
}

Edited by:

Hironori Sato, National Institute of Infectious Diseases, Japan

\section{Reviewed by:}

Masaru Yokoyama, National Institute of Infectious Diseases, Japan

Takao Masuda, Tokyo Medical and

Dental University, Japan

\section{*Correspondence:}

Akihide Ryo, Department of

Microbiology, Yokohama City University

School of Medicine, 3-9 Fuku-ura,

Kanazawa-ku, Yokohama, Kanagawa

236-0004, Japan.

e-mail:aryo@yokohama-cu.ac.jp

Induced pluripotent stem cells (iPSCs) are artificially derived from somatic cells that have been transduced with defined reprogramming factors. A previous report has indicated the possibility of using iPSCs as an immune stimulator to generate antigen-specific immunity. In our current study, we have investigated whether human iPSCs (hiPSCs) have the ability to enhance specific immune response against a human immunodeficiency virus type 1 (HIV-1) antigen in a xenogenic mouse model. Our results show that BALB/c mice immunized with hiPSCs transduced with an adenoviral vector encoding HIV-1 gp160 exhibited prominent antigen-specific cellular immune responses. We further found that pre-treatment of hiPSCs with ionizing radiation promotes the secretion of pro-inflammatory cytokines such as interleukin-1 alpha (IL-1 $\alpha)$, IL-12, and IL-18. These cytokines might promote the activation of antigen-presenting cells and the effective induction of cellular immunity. Our present findings thus demonstrate that a hiPSCs-based vaccine has the potential to generate cellular immunity against viral antigens such as HIV-1 gp160 in a xenogenic condition.

\section{Keywords: human induced pluripotent stem cells, ionizing radiation, pro-inflammatory cytokine, cellular immunity,} vaccine

\section{INTRODUCTION}

The latest estimate indicates that 33.4 million people are living with human immunodeficiency virus (HIV)/AIDS in 2009, and there are some 2.7 million new infections each year. Globally, HIV/AIDS is the leading cause of mortality among younger people and children where 2.0 million people die annually. The development of a safe and effective HIV/AIDS vaccine is required urgently to halt this epidemic. There is currently no effective AIDS vaccine available (McElrath and Haynes, 2010).

Current approaches in the development of HIV/AIDS vaccines have focused not on preventing infection itself, but on eliciting potent antiviral-specific $\mathrm{CD}^{+} \mathrm{T}$ cell [cytotoxic $\mathrm{T}$ lymphocyte $(\mathrm{CTL})]$ responses to limit viral replication (Yamamoto and Matano, 2008). This is in part because such an immune response contributes to non-progression of AIDS in people infected with HIV type 1 (HIV-1). It is thus desirable to develop a new vaccine vector system for generating effective CTL response against HIV-1.

Recently, reprogramming of human somatic cells into pluripotent embryonic stem (ES) cell-like cells, termed human induced pluripotent stem cells (hiPSCs) has been successfully achieved by the transduction of four defined transcription factors (Klf4, Oct4, Sox2, and c-Myc; Takahashi and Yamanaka, 2006; Takahashi et al., 2007; Park et al., 2008). This technology does not require embryos or oocytes, thereby facilitating the generation

Abbreviations: APC, antigen-presenting cell; CAG promoter, CMV enhancer $/ \beta$ actin promoter with $\beta$-actin intron; DsRed, Discosoma sp. red fluorescent protein; hiPSC, human induced pluripotent stem cell; HIV, human immunodeficiency virus; IL-1, interleukin-1; MHC, major histocompatibility complex; NF-kB, nuclear factor- $\kappa \mathrm{B}$. of individual-specific pluripotent stem cells that are valuable for personalized cell transplantation therapy without concern for immune rejection.

A recent report has demonstrated a new application of hiPSCs as an immune stimulator. Li et al. (2009b) demonstrated that the inoculation of human ES cells (ESCs) or hiPSCs can induce a protective immune response against murine colon cancer cells. It is considered that the pluripotent stem cell induces the immune response in vivo against colon cancer cells expressing oncofetal antigens that are present in normal stem cells (Li et al., 2009b). Moreover, other studies demonstrated that ESCs can produce pro-inflammatory cytokines and growth modulators following a treatment with ionizing radiation (Guo et al., 2006). We therefore hypothesized that irradiated hiPSCs expressing the HIV-1 antigen have a potency to induce the antigen-specific CTL response.

In our current study, we selected HIV-1 gp160 as a model antigen for evaluating the ability of hiPSCs as a vaccine vector. Our current results provide evidence for the capacity of hiPSCs to induce antigen-specific immunity against viral proteins.

\section{MATERIALS AND METHODS CELL CULTURE}

Human induced pluripotent stem cells (hiPSCs) were derived from adult human fibroblasts and were obtained from the RIKEN Bioresource Center (clone no. 201B7; Takahashi et al., 2007). The hiPSCs were cultured on plates coated with Matrigel (BD Biosciences, San Jose, CA, USA) and maintained in Knockout Dulbecco's modified Eagle's medium (Invitrogen, Carlsbad, CA, USA) supplemented with $20 \%$ Knockout SR (Invitrogen), 1\% GlutaMAX (Invitrogen), $100 \mu \mathrm{M}$ non-essential amino acids (Invitrogen), $50 \mu \mathrm{M} \beta$-mercaptoethanol, and $10 \mathrm{ng} / \mathrm{ml}$ human 
basic fibroblast growth factor (bFGF) and conditioned medium for mouse embryonic fibroblasts. MRC5 (human fetal lung fibroblasts) and RAW 264.7 (mouse monocyte/macrophage) cell lines were maintained in Dulbecco's modified Eagle's medium (DMEM; Wako, Osaka, Japan) supplemented with 10\% heat-inactivated fetal bovine serum.

\section{ADENOVIRUS VECTOR}

The adenovirus vectors expressing HIV-1 Rev and Env gp160 proteins (Ad-gp160) or Discosoma sp. red fluorescent protein (DsRed, Ad-Red) were generated as described previously (Abe et al., 2009). These adenoviral vectors are replication-defective recombinant adenovirus type 5 virus lacking the E1 and E3 regions (Mizuguchi and Kay, 1998). The expression of HIV-1 rev-env gp160, or DsRed was controlled using the CMV enhancer/ $\beta$-actin promoter with $\beta$-actin intron (CAG promoter). These vectors were propagated in HEK293 cells and purified by two repetitions of the $\mathrm{CsCl}$ method as described elsewhere (Lieber et al., 1996). The total concentration of virions in each preparation was calculated from the optical density at $260 \mathrm{~nm}\left(\mathrm{OD}_{260}\right)$, using the formula $1 \mathrm{OD}_{260}=1 \times 10^{12}$ viral particle $(\mathrm{vp}) / \mathrm{ml}$.

\section{ANTIBODIES AND IMMUNOBLOTTING}

HiPSCs were transduced with Ad-gp160 or Ad-Red at 3,000 vp/cell for $24 \mathrm{~h}$. The cellular lysates were then subjected to immunoblotting analysis with anti-HIV-1 gp120, anti-HIV-1 gp41 (AIDS Research and Reference Reagent Program, NIH, MD, USA), anti-Oct4 (C-10; Santa Cruz Biotechnology, Santa Cruz, CA, USA), anti-SOX2 (Millipore, Billerica, MA, USA), anti-phosphorylated IкB- $\alpha$ (Active Motif, Carlsbad, CA, USA), anti-IאB- $\alpha$ (Santa Cruz Biotechnology) or anti- $\beta$-actin monoclonal antibodies (AC-15; Sigma, Saint Louis, MO, USA). Immunoblotting images were acquired using LAS3000 (Fujifilm, Tokyo, Japan).

\section{FLOW CYTOMETRY ANALYSIS}

HiPSCs and MRC5 fibroblasts were harvested with 0.02\% EDTA in phosphate buffered saline (PBS), dissociated into single cells, and stained with the fluorescein isothiocyanate (FITC)-conjugated anti-HLA-ABC antibody (W6/32; BioLegend, San Diego, CA, USA). The cells were then analyzed by flow cytometry (FACSCantoII, BD Biosciences). For the evaluation of effector $\mathrm{CD}^{+} \mathrm{T}$ cells, peripheral blood mononuclear cells (PBMCs) from immunized mice were stained with phycoerythrin/cyanin7 (PE-Cy7)-conjugated anti-mouse CD62L (MEL-14; BioLegend) and FITC-conjugated anti-mouse CD8a antibodies (Ly-2; eBioscience, San Diego, CA, USA) without stimulation. The cells were then analyzed by flow cytometry (FACSCantoII). CD62 $\mathrm{L}^{-} \mathrm{CD}^{+} \mathrm{T}$ cells were defined as effector $\mathrm{CD}^{+} \mathrm{T}$ cells.

\section{ANIMAL IMMUNIZATION}

Generally, to estimate cell-based vaccine, $1 \times 10^{5}$ to $1 \times 10^{6}$ cells were inoculated into a mouse (Yoon et al., 2008; Li et al., 2009a; Senju et al., 2009). We thus introduced $5 \times 10^{5}$ cells in this immunization. We employed immunization protocol described as follows. Eight-week-old female BALB/c mice $\left(\mathrm{H}-2 \mathrm{D}^{\mathrm{d}}\right)$ were purchased from Japan SLC Inc. (Shizuoka, Japan) and housed in an animal facility. Under anesthesia, these mice were injected subcutaneously (s.c.) with $5 \times 10^{5} 15$ Gy-irradiated or formalin-fixed (2\%, $\left.15 \mathrm{~min}\right)$ hiPSCs expressing either DsRed or HIV-1 gp160, three times at 2- or 4 -week intervals. The study was approved by the Animal Administer Community of Yokohama City University.

\section{TETRAMER ASSAY}

Tetramer assay is a well-established way to examine the antigenspecific CTL in mouse models (Villacres et al., 2000; Hovav et al., 2007). Peptide 18 (p18: RGPGRAFVTI) derived from the V3 loop of HIV- $1_{\text {IIIB }}$ gp160 is an epitope recognized by CTL in the BALB/c mouse $\left(\mathrm{H}-2 \mathrm{D}^{\mathrm{d}}\right)$ model immunized with $\mathrm{HIV}-1_{\text {IIIв }}$ gp160 protein (Takahashi et al., 1988). H-2Dd/p18 tetramer was tetrameric $\mathrm{H}-2 \mathrm{D}^{\mathrm{d}}$ major histocompatibility complex (MHC) class I with the p18 labeled with phycoerythrin (PE) synthesized by NIH Tetramer 116 Core Facility (Atlanta, GA, USA). The number of $\mathrm{CD}^{+} \mathrm{T}$ cells expressing $\mathrm{T}$ cell receptor (TCR) that recognized $\mathrm{H}-2 \mathrm{D} / \mathrm{p} 18$ tetramer was examined by tetramer assay. Tetramer assays were performed using a $\mathrm{H}-2 \mathrm{D} / \mathrm{p} 18$ tetramer as previously described (Shimada et al., 2010). Briefly, PBMCs were isolated from immunized mice, and stained with a PE-conjugated $\mathrm{H}-2 \mathrm{D}^{\mathrm{d}} / \mathrm{p} 18$ tetramer and FITC-conjugated anti-mouse CD8a antibody. The cells were then analyzed by flow cytometry (FACSCantoII).

\section{INTRACELLULAR CYTOKINE STAINING ASSAY}

Intracellular cytokine staining (ICS) assays were performed as previously described (Shimada et al., 2010). Briefly, PBMCs were obtained from immunized mice, and the red blood cells were removed using lysing buffer (BD Bioscience). The lymphocytes were incubated with p18 (RGPGRAFVTI) from the V3 loop of HIV-1 ${ }_{\text {IIIв }}$ gp160 protein, and treated with BD GolgiStop (BD Bioscience) for $6 \mathrm{~h}$ at $37^{\circ} \mathrm{C}$. The cells were then stained with FITC-conjugated antimouse CD8a antibody, suspended in cytofix/cytoperm solution (BD Bioscience), and incubated with the PE-conjugated anti-mouse interferon-gamma (IFN- $\gamma$ ) antibody (XMG1.2; eBioscience). The cells were subsequently analyzed by flow cytometry (FACSCantoII) utilizing FACSDiva software.

\section{RT-PCR}

Total RNA was isolated by TRIzol reagent (Invitrogen), and reversetranscribed using ReverTra Ace (Toyobo, Osaka, Japan) in accordance with the manufacturer's instructions. The resulting cDNAs were amplified with Ex Taq DNA polymerase (Takara Bio, Otsu, Japan). To quantify the cytokine RNA levels, a SYBR premix Ex Taq II (Takara Bio) was used and analyses were performed using the ABI PRISM Sequence Detector System 7500 (Applied Biosystems, Foster City, CA, USA). The primer sets used for RT-PCR were as follows: interleukin (IL)-1 $\alpha$ : $5^{\prime}$-tcgagccaatgatcagtacc- $3^{\prime}$ and $5^{\prime}$-tggtctcactacctgtgatg- $3^{\prime} ; I L-18: 5^{\prime}$-gaccaagttctcttcattgacc- $3^{\prime}$ and $5^{\prime}$-tgtcctgggacacttctctg- $3^{\prime} ; I L-12 A: 5^{\prime}$-atgatggccetgtgccttag- $3^{\prime}$ and $5^{\prime}$-gtggcacagtctcactgttg- $3^{\prime} ; \quad$ Oct4: $\quad 5^{\prime}$-gagatatgcaaagcagaaacc- $3^{\prime}$ and $5^{\prime}$-cttcactgcactgtactcct- $3^{\prime}$; Nanog: $5^{\prime}$-atggttggagcctaatcagc- $3^{\prime}$ and $5^{\prime}$-tgttgtccttagcagccaag- $3^{\prime}$; hTERT: $5^{\prime}$-ccgtacatgcgacagttcg- $3^{\prime}$ and $5^{\prime}$-caagaaatcatccaccaaacg-3', and glyceraldehydes-3phospate dehydrogenase (gapdh): $5^{\prime}$-ccatggagaaggctgggg- $3^{\prime}$ and $5^{\prime}$-caaagttgtcatggatgacc- $3^{\prime}$. The $I L-1 \alpha, I L-18$, or $I L-12 A$ transcripts were normalized using the gapdh expression levels, and analyzed by the comparative $C_{\mathrm{T}}$ method. 


\section{ENZYME-LINKED IMMUNOSORBENT ASSAY}

The culture supernatants were collected from the irradiated hiPSCs at 24-h post-irradiation, centrifuged to remove cellular debris prior to filtration, and then subjected to enzyme-linked immunosorbent assay (ELISA) in accordance with the manufacturer's instructions (R\&D Systems, Minneapolis, MN, USA).

\section{ACTIVATION OF MOUSE SPLENOCYTES WITH hiPSCS}

Activation of mouse splenocytes with hiPSCs was detected by the co-culture system. Generally, the cell-to-cell co-culture system employs from 1:1 to 1:10 in the ratio of effector cells to target cells in number (Maitra et al., 2004; Hall et al., 2008). HiPSCs $\left(2 \times 10^{5}\right.$ cells) were co-cultured with mouse splenocytes $\left(2 \times 10^{6}\right.$ cells $)$ from naive mouse, at a ratio of $1: 10$. We employed the assay protocol described previously (Yoon et al., 2008). In brief, 200,000 hiPSCs were treated by either $15 \mathrm{~Gy}$-irradiation or $2 \%$ formalin solution for $15 \mathrm{~min}$, and then co-cultured or cultured in transwells (pore size, $0.4 \mu \mathrm{m}$ ) with $2 \times 10^{6} \mathrm{BALB} / \mathrm{c}$ mouse splenocytes for $40 \mathrm{~h}$. The splenocytes were then stained with FITC-anti-mouse CD11c (HL3; BD Bioscience) and PE-anti-mouse CD86 (PO.3; eBioscience) or PE-anti-mouse MHC class II antibodies (M5/114.15.2; eBioscience). The cells were analyzed under flow cytometry (FACSCantoII) utilizing FACSDiva version 6.1.2 software.

\section{MICROARRAY ANALYSIS}

Total RNAs for microarray analysis were isolated from hiPSCs cultured at $14 \mathrm{~h}$ post-irradiation with TRIzol (Invitrogen) and then analyzed using the Affymetrix GeneChip Human Genome U133 Plus 2.0 Array and GeneChip Scanner 3000. The gene expression levels were compared between irradiated hiPSCs and the nontreated control hiPSCs. Up-regulated genes showing at least a twofold change were identified using DNA MicroArray Viewer Software (Kurabo, Osaka, Japan).

\section{MEASUREMENT OF NUCLEAR FACTOR-KAPPA B ACTIVATION}

The culture supernatant was recovered from the irradiated hiPSCs at 24 -h post-irradiation. To neutralize IL- $1 \alpha$ in the culture supernatant, anti-human-IL-1 $\alpha$-neutralizing antibody (PeproTech, Rocky Hill, NJ, USA) or rabbit sera as control were added at 0.1 or $1 \mu \mathrm{g} /$ ml. Two hours after incubation, RAW 264.7 cells were incubated with the culture supernatants for $10 \mathrm{~min}$. Cell lysates were then immunoblotted with anti-phosphorylated I $\kappa$ B- $\alpha$ (Active Motif) or anti-IкB- $\alpha$ antibodies (Santa Cruz Biotechnology).

\section{STATISTICAL ANALYSES}

We performed the experiment with $n=3$. All values are expressed as mean \pm error. Statistical analyses were performed using Mann-Whitney's U-test with StatView 5.0 software (SAS Institute Inc., Cary, NC, USA), and $P<0.05$ was considered to be statistically significant.

\section{RESULTS}

\section{TRANSDUCTION OF UNDIFFERENTIATED HUMAN IPSCs WITH AN} ADENOVIRUS VECTOR EXPRESSING HIV-1 gp160

We initially investigated the efficient gene transfer into hiPSCs. Previous reports indicated that the CAG-promoter-driven adenoviral vector system can effectively transduce genes into hiPSCs without aberrant cell differentiation (Tashiro et al., 2010). We thus utilized a replication-defective human adenovirus type 5 vector encoding either HIV-1 gp160 (Ad-gp160) or DsRed (Ad-Red) under the control of the CAG promoter. We detected the successful expression of HIV-1 gp160 in hiPSCs by immunoblotting with either anti-HIV-1 gp41 or anti-HIV-1 gp120 antibodies (Figure 1A). Flow cytometric analysis further revealed that $>90$ and $60 \%$ of the transduced hiPSC population expressed DsRed and HIV-1 gp160, respectively (Figure 1B).

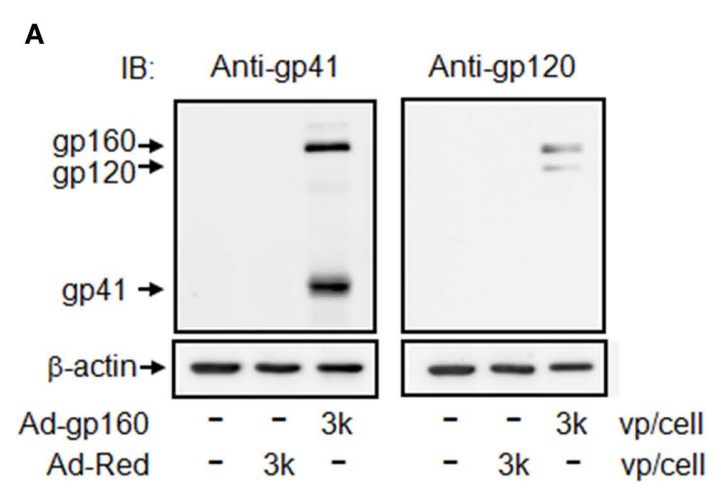

B
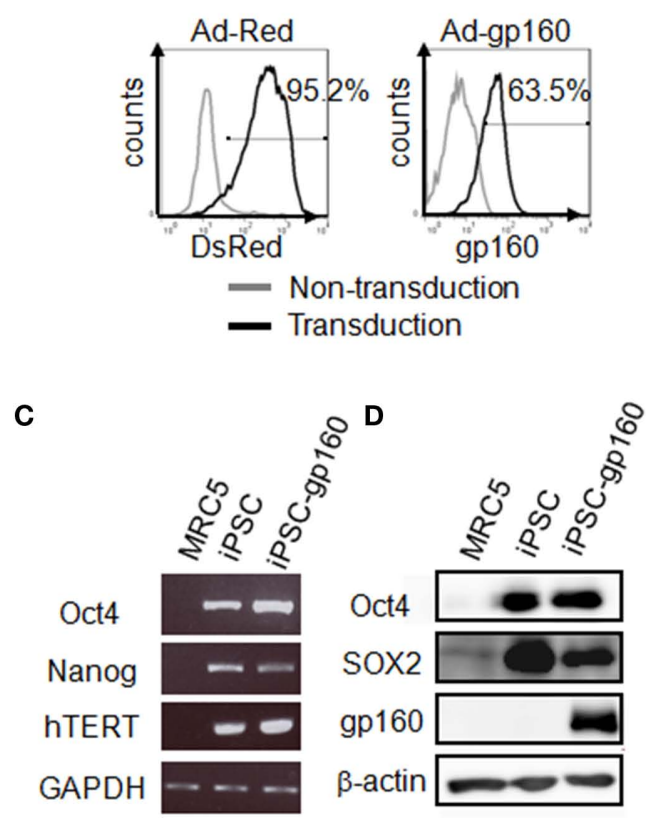

FIGURE 1 | Expression of HIV-1 gp160 in hiPSCs. (A,B) hiPSCs were transduced with adenoviral vectors encoding HIV-1 gp160 (3,000 vp/cell) or DsRed (3,000 vp/cell) for $24 \mathrm{~h}$. The HIV-1 gp160 protein and its cleaved product (gp41 and gp120) were detected by immunoblotting against anti-HIV-1 gp41 [(A) left] or anti-HIV-1 gp120 antibodies [(A) right]. $\beta$-actin was used as the internal control. The expression of DsRed or HIV-1 gp160 protein was measured by flow cytometry (B). The staining patterns of transduced (thick line) and non-transduced (thin line) cells are shown. Percentages of positive-cells after transduction with each adenoviral vectors are also indicated. (C) iPSC markers (Oct4, Nanog, and hTERT) and GAPDH mRNAs were detected in, MRC5 fibroblasts, non-transduced hiPSCs or Ad-gp160transduced hiPSCs at $24 \mathrm{~h}$ by RT-PCR. (D) The iPSC markers Oct4 and SOX2, and HIV-1 gp160 proteins were detected by immunoblotting of MRC5 fibroblasts, non-transduced hiPSCs, and Ad-gp160-transduced hiPSCs. 
We next addressed whether hiPSCs would retain their pluripotency following the transduction of exogenous genes. RT-PCR analysis revealed that the iPSC markers Oct4, Nanog, and hTERT were strongly expressed in the cells before and after the transduction of HIV-1 gp 160, but this was not the case in MRC5 fibroblasts (Figure 1C). Moreover, the protein expression of Oct4 and SOX2 was also detectable in Ad-infected hiPSCs during immunoblotting analysis (Figure 1D).

\section{IMMUNIZATION WITH hiPSCS AND THE IMMUNE RESPONSES GENERATED}

We investigated whether hiPSCs could induce cellular immune response against the viral antigen in a mouse model. Before immunization, flow cytometric analysis confirmed that hiPSCs expressed an extremely low level of MHC class I as compared with MRC5 fibroblasts on cell surfaces (Figure 2A), suggesting the minimum xenogenic reaction in a mouse inoculated with hiPSCs.

We next generated a formalin-fixed and irradiated hiPSCs-based vaccine expressing HIV-1 gp160. Because cell differentiation can be paralleled with cell proliferation as asymmetric cell division (Morrison and Kimble, 2006), the formalin-fixation or irradiation can abrogate both cell growth and differentiation (Figure 2B). To examine whether hiPSCs expressing HIV-1 gp160 could stimulate an immune response, we immunized immunocompetent BALB/c mice with either formalin-fixed or irradiated hiPSCs expressing HIV-1 gp160 (hiPSCs-gp160) or DsRed (hiPSCs-Red). These mice were boosted twice on days 15 and 40 (Figure 2C). In this protocol, the immunized mice did not show any clinical signs of adverse effects accompanied by xenogenic immune reaction such as skin rash, arthritis, or overt organ dysfunction (data not shown). On days $0,7,22$, and 47 after the first immunization, we performed the well-established $\mathrm{H}-2 \mathrm{D} / \mathrm{p} 18$ tetramer-binding assay. P18 (RGPGRAFVTI) is an epitope recognized by CTL in BALB/c mouse $\left(\mathrm{H}-2 \mathrm{D}^{\mathrm{d}}\right)$ model immunized with HIV-1 ${ }_{\text {IIIB }}$ gp160 protein (Takahashi et al., 1988). This assay measures $\mathrm{CD}^{+} \mathrm{T}$ cells recognizing the $\mathrm{H}-2 \mathrm{D}^{\mathrm{d}}$-restricted $\mathrm{p} 18$ derived from the V3 loop of the HIV-1 ${ }_{\text {III }}$ gp160 protein. At the same time, we conducted an ICS

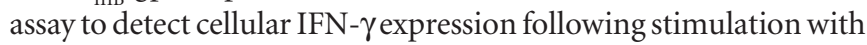
the $\mathrm{p} 18$. The results showed that $\mathrm{p} 18$ antigen-specific $\mathrm{CD} 8^{+} \mathrm{T}$ lymphocytes were substantially induced in mice injected with irradiated hiPSCs-gp160 as compared with other groups. It was statistically significant on Day 47 (Figure 2D). Although boost effects such as clonal expansion of p18-specific $\mathrm{CD}^{+} \mathrm{T}$ cells were not observed in mice after booster immunization with the hiPSC-based vaccine, antigen-specific IFN- $\gamma$-producing $C D 8^{+} \mathrm{T}$ lymphocytes were significantly increased up to 47 days in mice immunized with irradiated hiPSCs-gp160 further than the other groups (Figure 2E). Moreover, the proportion of total effector $\mathrm{CD}^{+} \mathrm{T}$ lymphocytes (CD62 $\mathrm{L}^{-} \mathrm{CD}^{+}$cells) was also found to be substantially increased by the immunization with irradiated hiPSCs-gp160 (Figure 2F).

We next examined the induction of HIV-1 gp160-specific antibodies in the sera from immunized mice by immunoblotting. We tested the immunoreactivity of the sera against cell lysates from either control 293T cells or those expressing HIV-1 gp160. We found that sera from mice immunized with formalin-fixed hiPSCsgp 160 contained antibodies that targeted the HIV-1 gp 160 protein and its cleavage product, gp41, whereas this was not the case for the sera from mice injected with irradiated hiPSCs (Figure 2G). Furthermore, the sera from mice immunized with formalin-fixed hiPSCs-gp160 but not with irradiated hiPSCs, contained antibodies against cell surface antigens of hiPSCs itself as revealed by flow cytometry (Figure $2 \mathbf{H}$ ).

These results indicate that formalin-fixed hiPSCs-gp160immunized mice were induced with antibodies against HIV-1 gp160 and against cell surface antigens of hiPSCs. The latter antibodies have the potential to rapidly eradicate hiPSCs-based vaccines during immunization in mice. Whereas, irradiated hiPSCs induced more antigen-specific $\mathrm{CD}^{+} \mathrm{T}$ cells than formalin-fixed hiPSCs and less antibody against hiPSCs.

\section{IRRADIATED hiPSCs STIMULATE CD11 ${ }^{+}$SPLENOCYTES THROUGH A CONTACT-INDEPENDENT MECHANISM USING SOLUBLE MOLECULES}

We next addressed whether irradiated hiPSCs could modulate the activity and function of immune cells such as antigen-presenting cells (APCs). Splenocytes from a BALB/c mouse were co-cultured with either formalin-fixed hiPSCs or irradiated hiPSCs, and then examined by flow cytometry for the expression of the activation marker CD86 or MHC class II in CD11 $\mathrm{c}^{+}$cells. Upon co-culture with irradiated hiPSCs, but not with formalin-fixed cells, the expression levels of both CD86 and MHC class II were significantly increased in the CD11 $\mathrm{c}^{+}$cell population (Figures $3 \mathrm{~A}, \mathbf{B}$ ).

To further investigate whether the stimulatory effects of irradiated hiPSCs upon CD11 $\mathrm{c}^{+}$cells were dependent on cell-to-cell interactions or soluble factors, transwell experiments were performed. HiPSCs and mouse splenocytes were separately cultured in the lower and upper chambers of a transwell, and then analyzed for the expression of activation markers on $\mathrm{CD} 11 \mathrm{c}^{+}$cell. Our results showed that these activation markers were indeed induced in the transwell system in a similar manner to the co-culture system (Figures 3C,D). We thus concluded that soluble factors from irradiated hiPSCs play a role in their stimulatory effects upon $\mathrm{CD} 11 \mathrm{c}^{+}$cells.

\section{INDUCTION OF PRO-INFLAMMATORY CYTOKINES IN hIPSCs BY RADIATION}

To identify the soluble factors that are secreted from irradiated hiPSCs, we performed a DNA microarray experiment to screen for ionizing radiation-induced factors in these cells. Total RNAs extracted from irradiated or untreated hiPSCs were subjected to DNA microarray analysis. Following data normalization, we selected genes that were induced more than two-fold upon exposure to ionizing radiation (Figure 4A). We found that multiple cytokines were up-regulated in hiPSCs by ionizing radiation. In particular, proinflammatory cytokine IL- $1 \alpha$ and T helper type 1 (Th1)-related cytokines IL-12A and IL-18 were prominently induced under these conditions. The expression of these cytokines was further analyzed by either conventional RT-PCR or quantitative RT-PCR (qPCR). Consistent with the microarray data, IL-1 $\alpha$, IL-12A, and IL-18 genes were found to be significantly increased at $24 \mathrm{~h}$ after irradiation (5.00-, 2.50-, and 2.46-fold, respectively; Figures 4B,C). Notably, the expression of these cytokines was not induced by an adenovirus infection (data not shown). We next investigated whether the cytokines production/induction was observed in MRC5 fibroblasts following ionizing irradiation. We found that there was little induction of IL-12A and IL-18 following the irradiation. Furthermore, 


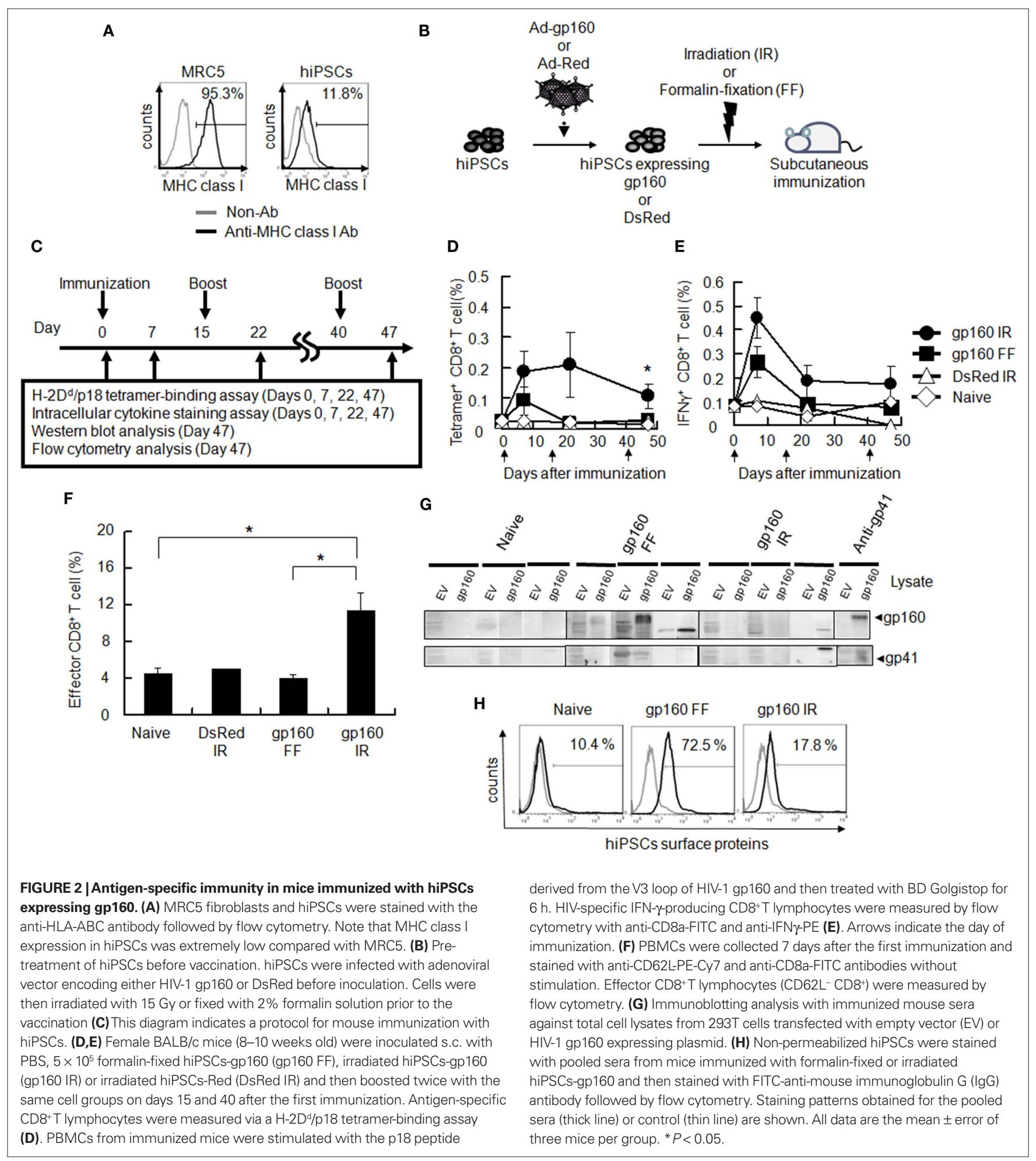

MRC5 fibroblasts did not express IL- $1 \alpha$ irrespective of the irradiation (Figure 4D). These results indicate that hiPSCs can efficiently produce these cytokines after irradiation.

We next examined the cytokine production levels from irradiated hiPSCs. Culture supernatants from these cells were collected and analyzed by ELISA. We found a substantial increase in the IL-1 $\alpha$ level upon irradiation (Figure 4E), whereas IL-12 and IL-18 were not detectable by ELISA (data not shown). We further analyzed the biological function of IL- $1 \alpha$ secreted from irradiated hiPSCs to mouse immune cells using a mouse monocyte/macrophage cell line RAW 264.7. Activation of RAW 264.7 was monitored by measuring of I $\mathrm{B}$ B- $\alpha$ phosphorylation. Cell supernatants from irradiated 


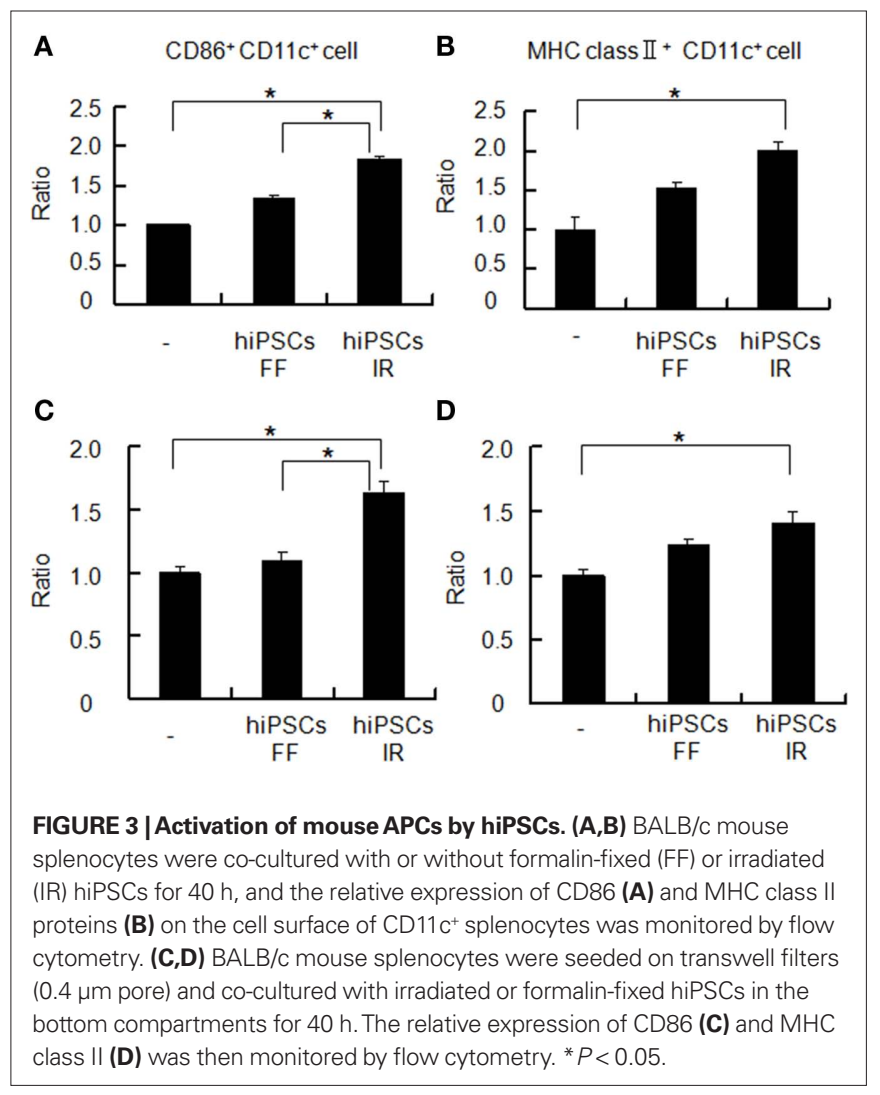

hiPSCs, but not control supernatant, significantly induced the phosphorylation of I $\mathrm{K}$ - $\alpha$, indicative of nuclear factor-kappa $\mathrm{B}$ $(\mathrm{NF}-\kappa \mathrm{B})$ activation (Figure $4 \mathrm{~F})$. A neutralizing antibody against IL- $1 \alpha$, but not control serum, was found to suppress this phosphorylation of IKB- $\alpha$ (Figure 4 F), demonstrating that IL- $1 \alpha$ from irradiated hiPSCs can activate NF- $\kappa \mathrm{B}$ signaling in RAW 264.7 cells. These results indicate that ionizing radiation can effectively induce the production of IL- $1 \alpha$ in hiPSCs for the activation of APCs and the antigen-specific immune responses.

\section{DISCUSSION}

Our present findings uncover a novel function of hiPSCs in the generation of viral antigen-specific immune responses as a cellbased vaccine. We found from our analyses that (1) Adenovirus system can effectively introduce exogenous viral antigen in hiPSCs without aberrant cell differentiation; (2) Radiation treatment can efficiently induce the cytokine production of IL-1 $\alpha$, IL-12, and IL-18; and (3) Inoculation of irradiated hiPSCs can enhance the antigen-specific cellular immune response even in xenogenic condition. These results indicate that hiPSCs can promote cellular immunity against an exogenously expressed viral antigen in a xenogenic condition.

We utilized the adenoviral vector system to express exogenous viral antigen in hiPSCs. Adenovirus has been shown previously to effectively transfer exogenous genes into hiPSCs (Tashiro et al., 2010) and we obtained a high efficiency in this regard without any effects upon cell differentiation. Moreover, $>75 \%$ of the cells survived the adenoviral transduction which typically causes a reasonable amount of cell death in primary cultures (data not shown). Thus the adenoviral vector system is suitable for use with hiPSCs owing to its high transduction efficiency and low cellular toxicity.

Our current immunization protocol demonstrated that the boost stimulation with irradiated hiPSCs-gp160 did not induce the clonal expansion of antigen-specific $\mathrm{CD}^{+} \mathrm{T}$ cells. Unlike the vaccine model with viral vectors, boost effects are usually not obvious in cell-based vaccines ( $\mathrm{Li}$ et al., 2009a). However, a boost stimulation can prolong the long-standing immune response against antigens. Consistently, our current hiPSCs vaccine system can induce relatively high levels of antigen-specific $\mathrm{CD}^{+} \mathrm{T}$ cells in the long term. This could be an advantage of the hiPSCs-based vaccine although further analytical and experimental studies are required to better understand and evaluate the vaccine system.

The immunization of mice with irradiated hiPSCs causes the induction of cellular immunity rather than humoral immunity, which is in contrast to the result of immunization with formalinfixed hiPSCs. Previous studies have suggested that Th1- and Th2-cells direct different immune response pathways (Kidd, 2003). Our present data indicates that irradiated hiPSCs may induce Th1-cytokines that contribute to the Th1 shift in immunized mice resulting in the induction of antigen-specific CTL. Furthermore, pre-treatment with ionizing radiation could reduce the generation of antibodies against the hiPSC vector itself. Thus, ionizing irradiation might be a proper procedure in the hiPSCs-based vaccine system.

In our current study, we evaluated the ability of hiPSCs to induce antigen-specific CTL as a cell-based vaccination in a xenogenic mouse model. In fact, a previous report presented by Li et al. (2009b) demonstrates that hiPSCs can produce a tumor antigen-specific immune reaction leading to a tumor rejection even in a xenogenic condition. It has been reported that MHC molecules could strongly induce xenogenic immune reactions. As we and others have revealed, hiPSCs express a very low level of MHC molecules on the cell surface (SuarezAlvarez et al., 2010). With this knowledge in hand, we decided to use the mouse model of HIV-1 gp160 to assess the ability of hiPSCs as a cell-based vaccine vector. However, it is necessary to investigate the involvement of the xenogenic condition in the antigen-specific immune reaction by the iPSCs-mediated vaccination.

We investigated the MHC-restricted immune response in the xenogenic mouse model in this report. It is likely that inoculated hiPSCs could be phagocytosed by mouse APCs such as dendritic cells followed by the antigen presentation and MHC-restricted immune activation in an immunized mouse. However, a previous report indicates that pluripotent stem cells such as ESCs or iPSCs may have a new system for antigen presentation since these cells express very low level of MHC molecules on their cell surface (Li et al., 2009b). Further experimental studies are necessary for a better understanding of the nature of iPSC as a new type of vaccine vector.

Cytokines are important signaling molecules that mediate communicative interactions both locally and between different cells in vivo. We have shown in our present study that irradiation of hiPSCs can selectively induce pro-inflammatory and Th1-related cytokines such as IL-1 $\alpha$, IL-12, and IL-18. Significantly, this was not the case in MRC5 fibroblasts. Cytokine production from irradiated hiPSCs might promote the activation of APCs thereby enhancing cellular immunity against loaded antigen. IL-1 $\alpha$ has been shown to be a potent pro-inflammatory cytokine and directly 


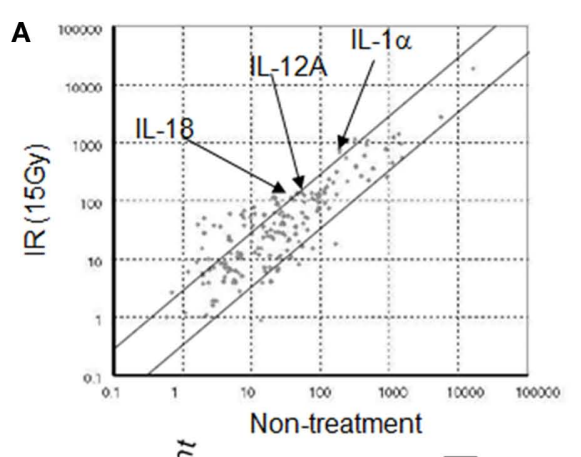

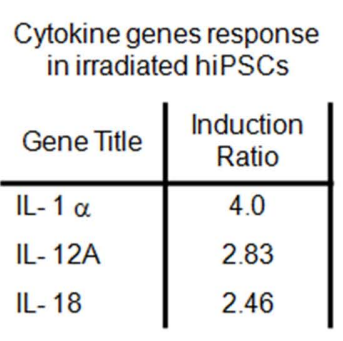
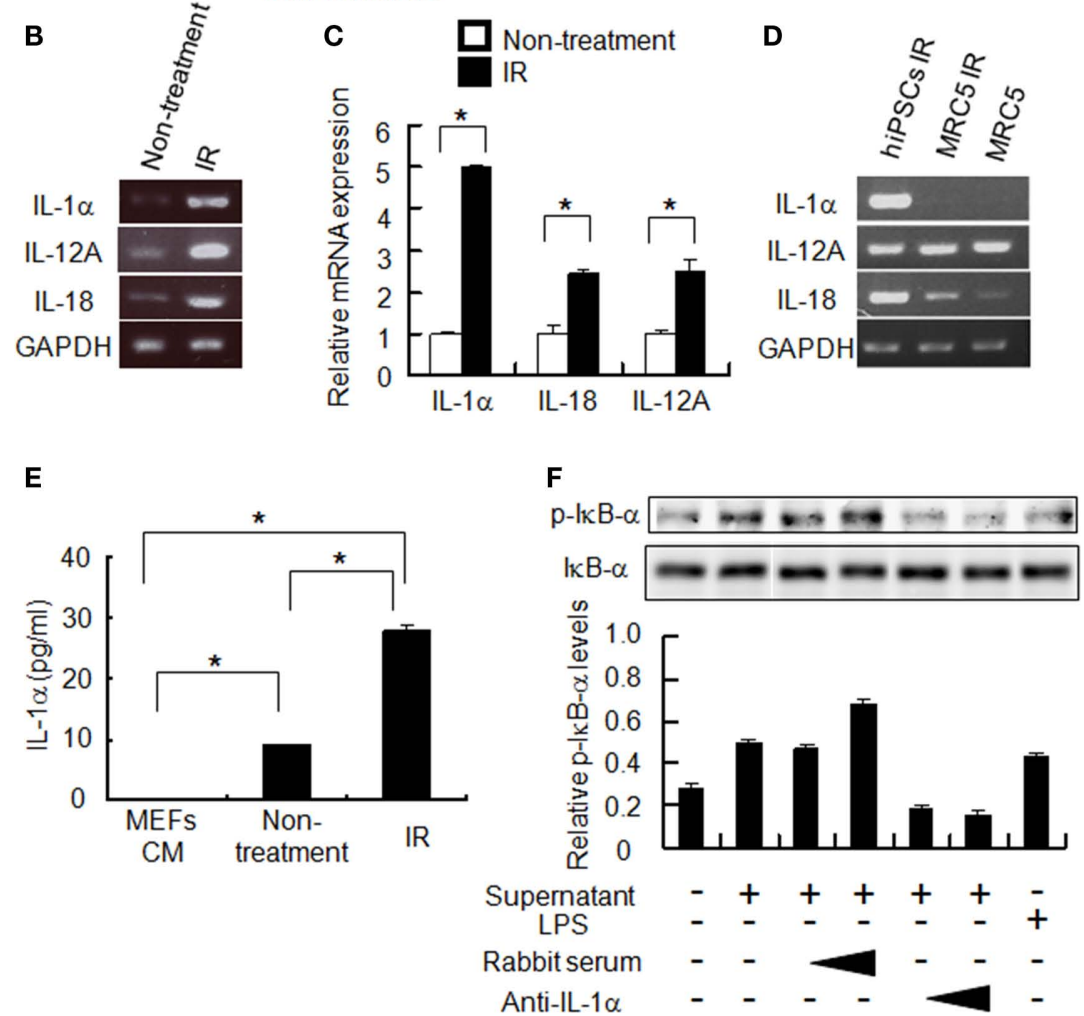

FIGURE 4 | Expression of immune-modulating cytokines in irradiated hiPSCs. (A) A scattered plot comparing the cytokine gene expression profiles of untreated and $15 \mathrm{~Gy}$-irradiated hiPSCs (IR). Arrows indicate the expression levels of IL-1 $\alpha, I L-12 A$, and IL-18. The lines indicate the two-fold changes between the samples. (B,C) HiPSCs were either treated or not treated with 15 Gy ionizing radiation. Twenty-four hours later, total RNAs were isolated from untreated or irradiated hiPSCs followed by conventional RT-PCR (B) or quantitative RT-PCR (qPCR) (C). Data is normalized to the gapdh mRNA levels and represent the mean \pm error of three independent experiments. ${ }^{*} P<0.05$. (D) MRC5

fibroblasts and hiPSCs were either untreated or treated with ionizing radiation
(15 Gy). After $24 \mathrm{~h}$, total RNAs were extracted and subjected to RT-PCR analysis for IL-1 $\alpha$, IL-12A, IL-18, and GAPDH. (E) Cell culture supernatants were collected from untreated or irradiated hiPSCs followed by IL-1 $\alpha$ ELISA. Conditioned media from mouse embryonic fibroblasts (CM-MEFs) was used as a control. (F) Filtered culture supernatants from hiPSCs were premixed with rabbit anti-human IL-1 $\alpha$-neutralizing antibody or control rabbit sera for $2 \mathrm{~h}$. RAW 264.7 cells were then exposed to these mixtures (culture supernatant and antibody) for 10 min before preparing whole cell lysates for immunoblotting analysis with either anti-phosphorylated $1 \kappa B-\alpha(p-\mid k B-\alpha)$ or anti-|кB- $\alpha$ antibodies. Lipopolysaccharide (LPS) was used as a positive control. up-regulate NF- $\mathrm{BB}$ signaling resulting in the activation of dendritic cells and macrophages (Ardeshna et al., 2000; Yoshimura et al., 2003; Weber et al., 2010). Although much progress has been made in dissecting the complexity of the cytokine network, the role of cytokines from pluripotent stem cells has not been well characterized. Our current results indicate however that the secretion of pro-inflammatory cytokines from hiPSCs might activate APCs via $\mathrm{NF}-\kappa \mathrm{B}$ activation to accelerate the induction of antigen-specific immune responses in vivo. Consistently, previous reports suggest the potent cross-reactivity of human IL- $1 \alpha$ and IL-18 to stimulate mouse immune cells whereas the stimulatory effect of human IL-12 p35/p40 hetero-dimer to mouse cells has shown itself to be very marginal (Libert et al., 1990; Schoenhaut et al., 1992; Kong and $\mathrm{Li}, 2002$ ). Although the precise molecular pathways underlying effective immune responses by hiPSCs have not been investigated, the irradiation-induced modulation of the cytokine expression in these stem cells might confer an effective adjuvant effect during the vaccination processes. 
In this report, we evaluated the immunogenicity of hiPSCs which expressed viral antigen in a mouse model. In the future, it is necessary to examine the potency of iPSCs in syn- or allogenic conditions using murine iPSCs. Additionally, the immunogenicity of non-iPSCs should be investigated in comparison with that of iPSCs. However, at least, even in the xenogenic condition, irradiated hiPSCs expressing HIV-1 gp160 protein could induce antigenspecific cellular immune response. Our current results thus provide evidence for the capacity of hiPSCs to induce antigen-specific immunity against the viral proteins.

\section{REFERENCES}

Abe, S., Okuda, K., Ura, T., Kondo, A., Yoshida, A., Yoshizaki, S., Mizuguchi, H., Klinman, D., and Shimada, M. (2009). Adenovirus type 5 with modified hexons induces robust transgenespecific immune responses in mice with pre-existing immunity against adenovirus type 5. J. Gene Med. 11, 570-579.

Ardeshna, K. M., Pizzey,A. R., Devereux, S., and Khwaja, A. (2000). The PI3 kinase, p38 SAP kinase, and NF-kappaB signal transduction pathways are involved in the survival and maturation of lipopolysaccharide-stimulated human monocyte-derived dendritic cells. Blood 96, 1039-1046.

Guo,Y., Graham-Evans, B., and Broxmeyer, H. E. (2006). Murine embryonic stem cells secrete cytokines/growth modulators that enhance cell survival/ anti-apoptosis and stimulate colony formation of murine hematopoietic progenitor cells. Stem Cells 24, 850-856.

Hall, B. M., Robinson, C. M., Plain, K. M., Verma, N. D., Carter, N., Boyd, R. A., Tran, G. T., and Hodgkinson, S. J. (2008). Studies on naive CD $4+$ CD25 + T cells inhibition of naive CD4 + CD25 - T cells in mixed lymphocyte cultures. Transpl. Immunol. 18, 291-301.

Hovav, A. H., Cayabyab, M. J., Panas, M. W., Santra, S., Greenland, J., Geiben, R., Haynes, B. F., Jacobs, W. R. Jr., and Letvin, N. L. (2007). Rapid memory CD8+ T-lymphocyte induction through priming with recombinant Mycobacterium smegmatis. J. Virol. $81,74-83$.

Kidd, P. (2003). Th1/Th2 balance: the hypothesis, its limitations, and implications for health and disease. Altern. Med. Rev. 8, 223-246.

Kong, J., and Li, Y.C. (2002). Upregulation of interleukin-18 expression in mouse primary keratinocytes induced to differentiate by calcium. Arch. Dermatol. Res. 294, 370-376.

Li, B., Simmons, A., Du, T., Lin, C., Moskalenko, M., Gonzalez-Edick, M., VanRoey, M., and Jooss, K. (2009a). Allogeneic GM-CSF-secreting tumor cell immunotherapies generate potent anti-tumor responses comparable to autologous tumor cell immunotherapies. Clin. Immunol. 133, 184-197.

Li, Y., Zeng, H., Xu, R. H., Liu, B., and Li, Z. (2009b). Vaccination with human pluripotent stem cells generates a broad spectrum of immunological and clinical responses against colon cancer. Stem Cells 27, 3103-3111.

Libert, C., Brouckaert, P., Shaw, A., and Fiers, W. (1990). Induction of interleukin 6 by human and murine recombinant interleukin 1 in mice. Eur. J. Immunol. 20, 691-694.

Lieber, A., He, C. Y., Kirillova, I., and Kay, M. A. (1996). Recombinant adenoviruses with large deletions generated by Cre-mediated excision exhibit different biological properties compared with first-generation vectors in vitro and in vivo. J. Virol. 70, 8944-8960.

Maitra, B., Szekely, E., Gjini, K., Laughlin, and Koc, O. N. (2004). Human mesenchymal stem cells support unrelated donor hematopoietic stem cells and suppress T-cell activation. Bone Marrow Transplant. 33, 597-604.

McElrath, M. J., and Haynes, B. F. (2010). Induction of immunity to human immunodeficiency virus type- 1 by vaccination. Immunity 33, 542-554.

Mizuguchi, H., and Kay, M. A. (1998). Efficient construction of a recombinant adenovirus vector by an improved in vitro ligation method. Hum. Gene Ther. 9, 2577-2583.

Morrison, S. J., and Kimble, J. (2006). Asymmetric and symmetric stem-cell divisions in development and cancer. Nature 441, 1068-1074.

Park, I. H., Zhao, R., West, J. A., Yabuuchi, A., Huo, H., Ince, T. A., Lerou, P. H., Lensch, M.W., and Daley, G. Q. (2008). Reprogramming of human somatic cells to pluripotency with defined factors. Nature 451, 141-146.

Schoenhaut, D. S., Chua, A. O., Wolitzky, A. G., Quinn, P. M., Dwyer, C. M., McComas, W., Familletti, P.C., Gately, M. K., and Gubler, U. (1992). Cloning and expression of murine IL-12. J. Immunol. 148, 3433-3440. M. J., Dennis, J., Haynesworth, S. E.,

\section{ACKNOWLEDGMENTS}

We thank the RIKEN BioResource Center (Ibaraki, Japan) for providing hiPSCs and MRC5 fibroblasts, the NIH Tetramer Core Facility (Atlanta, GA, USA) for providing tetramers and AIDS Research and Reference Reagent Program for antibodies. We also appreciate Dr. H Mizuguchi for donating the adenoviral vector, and Dr. M Shimada for critical reading of the manuscript. This work was partially supported by grants from Takeda Science Foundation, Uehara Memorial Foundation, and Kanagawa Nanbyo foundation to Akihide Ryo, and an Ishidsu Shun Memorial Scholarship to Shinji Yoshizaki.

Senju, S., Haruta, M., Matsunaga, Y., Fukushima, S., Ikeda, T., Takahashi, K., Okita, K., Yamanaka, S., and Nishimura,Y.(2009). Characterization of dendritic cells and macrophages generated by directed differentiation from mouse induced pluripotent stem cells. Stem Cells 27, 1021-1031.

Shimada, M., Yoshizaki, S., Jounai, N., Kondo, A., Ichino, M., Ryo, A., and Okuda, K. (2010). DNA vaccine expressing HIV-1 gp120/immunoglobulin fusion protein enhances cellular immunity. Vaccine 28, 4920-4927.

Suarez-Alvarez, B., Rodriguez, R. M., Calvanese, V., Blanco-Gelaz, M. A., Suhr, S. T., Ortega, F., Otero, J., Cibelli, J. B., Moore, H., Fraga, M. F., and Lopez-Larrea, C. (2010). Epigenetic mechanisms regulate $\mathrm{MHC}$ and antigen processing molecules in human embryonic and induced pluripotent stem cells. PLoS ONE 5, e10192. doi: 10.1371/journal.pone.0010192

Takahashi, H., Cohen, J., Hosmalin, A., Cease, K. B., Houghten, R., Cornette, J. L., DeLisi, C., Moss, B., Germain, R. N., and Berzofsky, J. A. (1988). An immunodominant epitope of the human immunodeficiency virus envelope glycoprotein gp160 recognized by class I major histocompatibility complex molecule-restricted murine cytotoxic T lymphocytes. Proc. Natl. Acad. Sci. U.S.A. 85, 3105-3109.

Takahashi, K., Tanabe, K., Ohnuki, M., Narita, M., Ichisaka, T., Tomoda, K., and Yamanaka, S. (2007). Induction of pluripotent stem cells from adult human fibroblasts by defined factors. Cell 131, 861-872.

Takahashi, K., and Yamanaka, S. (2006). Induction of pluripotent stem cells from mouse embryonic and adult fibroblast cultures by defined factors. Cell 126, 663-676.

Tashiro, K., Kawabata, K., Inamura, M., Takayama, K., Furukawa, N., Sakurai, F., Katayama, K., Hayakawa, T., Furue, M. K., and Mizuguchi, H. (2010). Adenovirus vector-mediated efficient transduction into human embryonic and induced pluripotent stem cells. Cell. Reprogram. 12, 501-507.
Villacres, M. C., Zuo, J., and Bergmann, C. C. (2000). Maintenance of CD8(+) T-cell memory following infection with recombinant sindbis and vaccinia viruses. Virology 270, 54-64.

Weber, A., Wasiliew, P., and Kracht, M. (2010). Interleukin-1 (IL-1) pathway. Sci. Signal. 3, cml.

Yamamoto, H., and Matano, T. (2008). Anti-HIV adaptive immunity: determinants for viral persistence. Rev. Med. Virol. 18, 293-303.

Yoon, T. J., Kim, J. Y., Kim, H., Hong, C., Lee, H., Lee, C. K., Lee, K. H., Hong, S., and Park, S. H. (2008). Anti-tumor immunostimulatory effect of heatkilled tumor cells. Exp. Mol. Med. 40, 130-144.

Yoshimura, S., Bondeson, J., Brennan, F. M., Foxwell, B. M., and Feldmann, M. (2003). Antigen presentation by murine dendritic cells is nuclear factor-kappa B dependent both in vitro and in vivo. Scand. J. Immunol. 58, 165-172.

Conflict of Interest Statement: The authors declare that the research was conducted in the absence of any commercial or financial relationships that could be construed as a potential conflict of interest.

Received: 06 January 2011; paper pending published: 11 January 2011; accepted: 04 February 2011; published online: 22 February 2011.

Citation: Yoshizaki S, Nishi M, Kondo A, Kojima Y, Yamamoto $N$ and Ryo $A$ (2011) Vaccination with human induced pluripotent stem cells creates an antigenspecific immune response against HIV-1 gp160. Front. Microbio. 2:27. doi: 10.3389/ fmicb.2011.00027

This article was submitted to Frontiers in Virology, a specialty of Frontiers in Microbiology.

Copyright (c) 2011 Yoshizaki, Nishi, Kondo, Kojima, Yamamoto and Ryo. This is an open-access article subject to an exclusive license agreement between the authors and Frontiers Media SA, which permits unrestricted use, distribution, and reproduction in any medium, provided the original authors and source are credited. 